\title{
Die Bestimmung der Wasserstoffionenkonzentration in der Oto-Rhino-Laryngologie.
}

\author{
Von \\ H. Tsukamoto, K. Yoshitake u. D. Kawahara. \\ (Aus der oto-rhino-laryngologischen Abteilung des Dairen-hospitals.) \\ (Erste Mitteilung.)
}

\section{Einleitung.}

Seit Schade im Gebiete der Entzündung durch Messung der Wasserstoffionenkonzentration am Lebenden sehr erhebliche Abweichungen von der Norm feststellte, erregten die Untersuchungsmethoden der Wasserstoffionenkonzentration grösste Aufmerksamkeit in vielen Gebieten der Medizin und wurden sehr häufig angewendet, und heute muss diese Hyperionie der Entzündung gemeinsam mit der osmotischen Hypertonie usw. als Kardinalsymptom des physikalisch-chemischen Entzündungsbegriffs angesehen werden. Doch liegen genaue Messungen der PH-Werte in unserum Spezialfach meines Wissens ausser der von Marchionini und Mittermaier an den Schleimhäuten der oberen Luftwege bisher fast nie vor. Daher möchten wir über die Untersuchungen berichten, die sich damit beschäftigt haben, die Wasserstoffionenkonzentration mittelst der Chinhydronmethode nach Bïlmann an entzündlichen Produkten bes. an Eiter in unserem Spezialfach zu bestimmen.

A) PH-Werte des Eiters der akuten Peritonsillitiden.

I) Untersuchungsmethode.

Zur Bestimmung der Wasserstoffionenkonzentration stehen zurzeit zwei prinzipiell verschiedene W'Wege zur Verfügung, d.h. die elektrische Methode und die kolorimetrische mit Hilfe von bestimmten Indikatoren. Wir arbeiteten mit der elektrischen Methode besonders mit der Chinhydronkettenmethode von Bïlinann, welche mit der älteren Wasserstoffgasmethode solange in guter Übereinstimmung ist, als die Untersuchungslösung nicht 
eine Alkalität von $\mathrm{PH}-8$, o oder eine Salzkonzentration von ca. $\mathrm{m} / 5$ überschreitet. Aber physiologische sowie pathologische Flüssigkeiten z.B. Eiter liegen innerhalb dieser Grenzen; sie sind nicht so salzreich oder alkalisch, dass sie deshalb mit Chinhydron abweichende Ergebnise liefern. Bei unseren Untersuchungen haben wir folgende Kette angewendet.

Normalelektrode/ Standardlösung/ Mit Chinhydron gesättigte Untersuchungslösung. Als Normalelektrode haben wir ein Gemisch von einem Teil der I/Io Normalsalzsäure mit neun Teilen der I/Io Normal-KCLLösung angewendet, das mit Chinhydron, das wir im Dairenhospital fabrizierten, gesättigt und in welches Gemisch ein Platindraht eingeführt wurde. Als Standardlösung haben wir gesättigte KCL-Lösung angewendet. Nunmehr ist die Wasserstoffionenkonzentration dẹ Lösung der Normalelektrode gleich $\mathrm{PH}^{\prime}-2, \mathrm{O} 4$ bei $\mathrm{I}^{\circ} \mathrm{C}$; daher wird $\mathrm{PH}^{2}$ der zu untersuchenden Lösung aus folgender Gleichung ausgerechnet;

$$
\mathrm{PH}^{2}=2,04+\frac{\pi}{0.0577+0.0002(\mathrm{t}-\mathrm{10})}
$$

Dabei zeigt $\pi$ die Differenz zwischen dem Potential, das an der Berührungsfläche der zu untersuchenden Lösung mit der Standardlösung entsteht, und demjenigen, welches das bekannte Potential der Lösung der Normalelektrode mit gesättigter KCL-Lösung ist, und t zeigt die Zimmertemperatur in $\mathrm{C}^{\circ}$ an. Die Messung selbst geschehen mit Hilfe der Kompensationsausschaltung nach Poggendorf, Du Bois Raymond in der Gaskettenapparatur von Michaelis.

\section{Bemerkungen.}

Wohl ist die Chinhydronmessung gegenüber der Messung mit der Wasserstoffgaselektrode einfacher und nicht so zeitraubend, weil dabei der Stillstand des Spiegelgalvanometers immer ausgeprägt rascher als bei der Gaskettenmethode hervorgebracht, wird, aber, sie hat den einen Nachteil, dass sie die Kohlensäurespannung des Gewebes unberücksichtigt lässt. Für das Blut, Gewebssaft, Eiter usw. beträgt normalerweise diese Spannung 5,6 Vol.-\%., und wenn der Eiter, so wie es bei unseren Versuchen notwendig war, aus der peritonsillären Abszesshöhle entfernt wird, so tritt sehr rasch eine Abdunstung von Kohlensäure ein. Trotzdem findet bei der Chinhydronmessung keine Zuführung von Kohlensäure statt. Deswegen werden 
wir dabei stets etwa um PH-o,34 weniger saure Werte erhalten als bei der Messung mit der Wasserstoffgaselektrode. Aber eine rasche Messung der PH-Werte nach Entnahme des Eiters war uns nicht möglich, da wir in unserer Abteilung keine Gaskette besitzen und wir die Apparatur in der ziemlich entfernten zahnärztlichen Abteilung benutzen mussten. Daher wurden die Messungen möglichst genau zwei Stunden nach dem Auswerfen begonnen, um den Fehler der nachträglichen Reaktionsveränderungen infolge der Kohlensäureabdunstung möglichst gleichartig zu gestalten. Mit dieser Methode liessen sich bei Wiederholungen gut reproduzierbare Werte erzielen.

\section{2) Untersuchungsflüssigkeit.}

Als Untersuchungsmaterial haben wir den Eiter bei den 23 Fällen der Peritonsillitis purulenta acuta dadurch gewonnen, dass wir den hyperämisch, ödematös angeschwollenen Weichergaumen inzisierten. Dann haben wir ca. $0,5 \mathrm{cc}$ des ausgeworfenen Eiters in 2,5 cc destilliertes Wasser beigemengt und mit einem Glasstab vorsichtig umgerührt. Dabei wurde stets darauf geachtet, dass diese Flüssigkeit nicht mit dem Blut vermengt werde, weil die Eiterreaktion sich nicht wenig nach der alkalischen Seite ändern könnte. Wo das vorkam, und es liess sich nicht immer vermeiden, wurde der betreffende Fall ausgeschieden, darauf haben wir die Flüssigkeit filtriert, und diese Flüssigkeit dann als Untersuchungsmaterial verwendet, nachdem wir sie zuvor mit Chinhydron sich hatten sättigen lassen.

\section{Bemerkungen.}

r) Zunächst war nun noch die wichtige Frage bezüglich der Methode, ob die Wasserstoffionen des Eiters durch die Verdünnung mit dem destilliertem Wasser beeinflusst werden $\mathrm{zu}$ beantworten. Wenn man eine reine Säure verdünnt, so sinkt mit der fortschreitenden Verdünnung ihre Wasserstoffionenkonzentration, aber der experimentelle Vergleich von Eiteraufschwemmungen in verschiedenen Verdünunngen ergab, dass der Fehler bei der PH-Bestimmung höchstens O,I bis 0,2 beträgt, und damit war dann auch die Zuverlässigkeit des Verfahrens in dieser Richtung gesichert.

2) Zugleich aber wurde auch ein weiteres Bedenken erledigt. Das destilliẹtẹ Wassẹ ișt națürliç nicht neutral, wiẹ das thẹoretisch zu erwarten 
ist. Reines Wasser, das absolut neutral reagiert, wurde ganz ausserordentlich selten hergestellt, weil es sehr schwer ist aus dem Wasser die Kohlensäure, die es aus der Luft aufnimmt, zu èntfernen. Wegen dieser Beimengung reagiert das gewöhnliche destillierte Wasser stets sauer, aber die Störung der Untersuchungsergebnisse durch die Kohlensäure des destillierten Wasser war sehr gering und daher zu vernachlässigen.

3) Drittens müssen wir immer als Glasinstrumente, z.B. Glasbecher, Glasrohr usw., möglichst nur solche aus Kieselglas benutzen, da bekanntermassen bei der Verwendung von Instrumenten aus anderen Glasarten die Reaktion der Untersuchungsflüssigkeit mehr oder weniger nach alkalischer Seite verschoben sein kann.

3) Untersuchungsergebnisse und Kritisches.

Wir haben die Untersuchungsergebnisse in der Tabelle I. zusammengestellt, so dass sie leicht zu übersehen sind. So haben die PH-Werte des Eiters bei 23 Peritonsillitiden verschiedene Grade, die zwischen dem Maximalwert $\mathrm{PH}-7, \mathrm{I} 8$ und dem Minimalwert PH-6,39 liegen, und ibr Durchschnittswert ist PH-6,70. Die PH-Werte von 5 Fällen darunter fallen fast in den Neutralbereich (von PH-6,8 bis zu PH-7,r), aber die PH-Werte weiterer I6 Fälle liegen im Gebiete der akuten Entzündung von Schade d.h. unterhalb PH-6,8, und schliesslich sind die PH-Werte bei den übrigen 2 Fälle oberhalb $\mathrm{PH}-7, \mathrm{I}$.

Hiersei noch ein Wort hinzugefügt. Es darf angenommen werden, dass die Rachenschmerzen bei der Peritonsillitis pupulenta mehr in inniger Beziehung zu dem PH-Werte des peritonsillären Eiters stehen müssen als zu den mechanischen Dehnungen der umgebenden Gewebe durch den peritonsillären Eiter, denn erstens hatten die gesamten 23 Fälle fast gleichgradige Anschwellungen der Gaumenbögen usw, und zweitens hatten die 7 Fälle im Neutralbereich geringere Rachenschmerzen als die weiteren I6 Fälle, welche niedere PH-Werte unterhalb PH-6,8 hatten.

\section{B) PH-Werte der Ohrensekrete der akuten purulenten Otitiden.}

Zunächst stellten wir die Bestimmungen der Wasserstoffionenkonzentration der Ohrensekrete bei.22 Fällen der Otitis media purulenta acuta an 
ihren verschiedenen Krankheitstagen fest, daher wollen wir in diesem Abschnitte ihre Untersuchungsergebnisse kurz berichten.

Als Untersuchungsflüssigkeit haben wir die Ohrensekrete in der Weise gewonnen, dass wir die äusseren Gehörgänge der betreffenden Patienten mehrmals mit destilliertem Wasser ausspülten und dann mit aseptischer entfetteten Watte reinigten und drei Stunden danach mit schmalen Kieselglasrohr ca. o, I cc. den aus dem Perforationsloch des Trommelfells hervorquwollenden Eiter heraussaugten, dann 'ihm ca. I,o cc. destilliertes Wasser beimengten, diese Mischung mit einem Glasstab umrührten und endlich sie sich mit Chinhydron sättigen liessen. Bei dieser Gelegenheit ist zu achten, dass die Zähigkeit der Ohrensekrete häufig das Beimengen mit destillietem Wasser, wie es unsere Methode erfordert, fast unmöglich macht und deswegen solche Ohrensekrete zu diesem Experimente nicht verwendet werden können. Wo das vorkam, und es liess sich nicht immer vermeiden, wurde der betreffende Fall ausgeschieden.

\section{Untersuchungsergebnisse und Kritisches.}

Wir haben die Untersuchungsergebnisse in den folgenden (Tabellen 2 u. 3) zusammengestellt, dass sie zu leicht übersehen sind. Dabei zeigt $D$ den Krankheitstag nach der spontanen Perforation oder Parazenthese des Trommelfells, $\mathrm{T}$ die Zimmertemperatur in $\mathrm{C}^{\circ}$ an.

I) Im allgemeinen vermehrten sich die $\mathrm{PH}$-Werte der Ohrensekrete bei allen Fällen der akuten putriden Otitiden ausser in zwei Fällen (Fall. I und Fall. 9) mit dem Verlauf der Krankheitstage. Aber ausnahmsweise nahm allmählich der PH-Wert des Fall. 9 während der I 5 Krankheitstage nach der Perforation usw. des Trommelfells bis zu PH-6,86 zu, danach verminderte er sich allmählich und schliesslich zeigte der betreffende Fall am 35ten Tag PH-6,38 sowie eine auftallende Verschlimmerung des allgemeinen und lokalen Status und zuletzt wurde er am 36ten Tag als Antrozellulitis purulenta akuta antrotomiert.

In ähnlicher Weise ging es bei Fall. I zu. Der PH-Wert des Ohrensekrets nahm am igten Tag nach der Perforation des Trommelfells bis PH-7,O4 zu, am 35ten Tag nahm er bis PH-6,78 ab und der betreffende Fall wurde auch am 42ten Tag als Mastoidzellenempyem antrotomiert. 
2) Wir können im allgemeinen die $\mathrm{PH}-$ Werte in allen Fällen der akuten Otitiden, ausser in zwei Fällen (Fall. I und Fall. 9), an ihren dritten Krankheitstagen in drei Gruppen einteilen.

a). Die erste Gruppe (Fall. 7, I4, 8, 19, I8, 21, 6, I0 und I7) hatte verschiedene $\mathrm{PH}-$ Werte unter $\mathrm{PH}-6,60$. Bei vier Fällen (Fall. 6, 21, i7 und 8) vermehrten sich allmählich ihre $\mathrm{PH}$-Werte und während unserer Beobachtungszeit d.h. in 35 Tagen trat bei den Ohrensekreten der drei Fälle (Fall. 6, 2I und 8) im Neutralbereich im Stillstand ein, während das Sekret beim Fall. I7 im sauren Gebiet zum Stillstand kam. Die PH-Werte nahmen in zwei Fällen (Fall. I 8 und Fall. 19) mit dem Verlauf der Krankheitstage 'zu und am Ende unserer Beobachtungszeit lagen sie noch innerhalb des sauren Gebietes. Die PH-Werte der anderen zwei Fälle (Fall. 7 und IO) vermehrten sich viel rascher und am Ende unserer Beobachtungszeit lagen sie im Neutralbereich, und der PH-Wert des Fall. I 4 erhöhte sich ausgeprägt rascher und er lag zum Ende der Beobachtungszeit im alkalischen Gebiet.

b). Die zweite Gruppe (Fall. I2, I3, 3, 22, I I, 20 und I 5) hatte verschiedene $\mathrm{PH}-$ Werte zivischen $\mathrm{PH}-6,60$ und $\mathrm{PH}-6,80$. Unter diesen Fällen bei drei Fällen (Fall. I2, I 3 u. 3) wieder erhöhten sich allmählich ihre PH-Werte, und während unserer Beobachtungszeit standen die Ohrensekrete aller drei Fälle im Neutralbereich still. Die PH-Werte bei drei Fällen (Fall. 20, 22 und I I) stiegen mit dem Verlauf der Krankheitstage und am Ende unserer Beobachtungszeit waren sie im Neutralbereich angelangt. Der PH-Wert des Fall I 5 erhöhte sich allmählich und am Ende der Beobachtungszeit war er im alkalischen Gebiet.

c). Die dritte Gruppe (Fall. 2, 5, 4 und 16) hatte verschiedene PH-Werte zwischen PH-6,80 und PH-7,ro. Die PH-Werte bei zwei Fällen (Fall. 2 und Fall. 5) nahmen allmählich $\mathrm{zu}$ und waren am Ende der Beobachtungszeit im Neutralgebiet. Die PH-Werte der anderen zwei Fälle (Fall. 4 und I6) vermehrten sich auch allmählich und waren am Ende der Beobachtungszeit im alkalischen Gebiet.

d). Schliesslich fügen wir noch eine Bemerkung. Die Verschiebungen der PH-Werte der Ohrensekrete erfolgten in allen Fällen der akuten putriden Otitiden ausser in zwei Fällen (Fall. I und Fall. 9) mit dem Verlauf 
der Krankheitstagen durchschnittlich, wie die punktierte Linie in der Tabelle 3 zeigt; am 3 ten Tag nach der spontanen. Perforation oder Parazenthese des Trommelfells PH-6,60, am 7ten Tag PH-6,66, am I Iten Tag PH-6,73, am I5ten Tag PH-6,77, am Igten Tag PH-6,85, am 23ten Tag PH-6,88, am 27ten Tag PH-6,92, am 3iten Tag PH-6,97, am 35ten Tag PH-6,99.

\section{Schluss.}

Wir haben durch die Bestimmung der Wasserstoffionenkonzentration mittelst der Chinhydronkettenmethode folgende Tatsachen wahrgenommen; I) Die PH-Werte des Eiters bei 23 Peritonsillitiden haben verschiedene Werte zwischen dem Maximalwert PH-7, I 8 und dem Minimalwert PH-6,39, und ihr Durchschnittswert ist PH-6,70. 2) Die PH-Werte der Ohrensekrete bei 20 akuten putriden Otitiden vermehren sich mit dem Verlauf der Krankheitstage; durchschnittlich sind die PH-Werte am 3ten Tag nach der spontanen Perforation oder Parazenthese des Trommelfells PH-6,60, am 7 ten Tag PH-6,66, am IIten Tag PH-6,73, am I5ten Tag PH-6,77, am I9ten Tag PH-6,85, am 23ten Tag PH-6,88, am 27ten Tag PH-6,92, am 3 Iten Tag PH-6,97 und am 35ten PH-6.99. 3) Bei zwei Fällen der akuten putriden Otitiden wandern die PH-Werte einmal im Verlauf der Krankheitstage nach alkalischer Seite. Darauf sinken sie herab in das saure Gebiet, wenn die beide Fälle mit Mastoidzellenempyem auftreten.

\section{Literatur.}

1) K. Rohlde ; Entzündung und Wasserstuffionenkonzentration. Deut. med. Wcchenschr, Jahrg. 58, 352. (1927). $\quad$ 2) W. v. Gaza u. B. Brandi; Beziehungen zwischen Wasserstoffionenkonzentration und Schmerzempfindung. Klin. Wochenschr, Jahrg. 5, 1123. (1926):

3) Hermann Gänssle; Die Wasserstoffionenkonzentration im Scheidensekret. Arch. f. Gynäk, 123, 602. (1924). $\quad$ 4) R. Mittermaier u. A. Marchionini; Messungen der Wasscrslofflonenkonzentration an den Schleimhäuten der oberen Luftwege. Zeitschr. f. H. N. u. O., 20, 253. (1928). 5) A. Itano \& K. Hosoda ; Investigation on the Biilmann's QuinhydronElectrode for the Determination of Hydrogen-Ion Concentration. The agricultural chemical society of Japan, 2, 13. (1926). $\quad$ 6) K. Tanaka u. S. Tamamura; Die Bestimmung der Wasserstoffzahl mittels der Chinhydronkette. The Jounal of Oriental Medicine, 5, 416. (1926). $\quad$ 7) Leonor Michaelis; Die Wasserstoffionenkonzentration. (1922). Ferdinand Hoff; Über die Wasserstoffionenkonzentration im Sputum. Klin. Wochenschr., Jahrg. 4, 1059. (1925). $\quad 9)$ Ernst Mislowitzer; Zur Messung der PH von Plasma, Serum und Blut mit der Chinhydronelektrode. Klin. Wochschr, Jahrg 5, 1863. (1926). 
Tabelle 1 .

\begin{tabular}{|c|c|c|c|c|c|c|c|}
\hline Fall & $\begin{array}{l}\text { Zimmer- } \\
\text { temperatur } \\
\text { in }{ }^{\circ} \mathrm{C}\end{array}$ & Millivolt & , $\mathrm{PH}$ & Fall & $\begin{array}{l}\text { Zimmer- } \\
\text { temperatur } \\
\text { in }{ }^{\circ} \mathrm{C}\end{array}$ & Millivolt & $\mathrm{PH}$ \\
\hline I & 24 & 274,0 & 6,69 & $\mathbf{r}_{3}$ & 23 & 261,0 & 6,48 \\
\hline 2 & 25 & 262,0 & 6,45 & I4 & 25 & 286,0 & 6,87 \\
\hline 3 & 27 & 274,0 & 6,64 & I 5 & 25 & 293,0 & 6,99 \\
\hline 4 & 24 & 279,0 & 6,77 & 16 & 24 & 299,0 & $7, \mathrm{II}$ \\
\hline 5 & 24 & 281,0 & $6,8 \mathrm{I}$ & I7 & 23 & 277,0 & 6,62 \\
\hline 6 & 23 & 290,0 & 6,98 & I8 & 25 & $3^{\circ}, 0$ & 7,58 \\
\hline 7 & 23 & 260,0 & 6,49 & 19 & 26 & 270,0 & 7,59 \\
\hline 8 & 26 & $25^{8,0}$ & 6,39 & 20 & 25 & 274,0 & 6,67 \\
\hline 9 & 22 & 277,0 & 6,77 & $2 \mathrm{I}$ & 24 & 267,0 & 6,57 \\
\hline IO & 23 & 276,0 & 6,74 & 22 & 25 & $28_{3}, 0$ & 6,82 \\
\hline II & 24 & 275,0 & 6.70 & 23 & 27 & 266,0 & $6,5^{\mathrm{r}}$ \\
\hline 12 & 26 & 260,0 & 6,42 & & & & \\
\hline
\end{tabular}

Tabelle 2.

\begin{tabular}{|c|c|c|c|c|c|c|c|}
\hline \multicolumn{4}{|c|}{ Fall. I. } & \multicolumn{4}{|c|}{ Fall. 3 . } \\
\hline $\mathrm{D}$ & $\mathrm{T}$ & Millivolt & $\mathrm{PH}$ & $\mathrm{D}$ & $\mathrm{T}$ & Millivolt & $\mathrm{PH}$ \\
\hline 3 & $25^{\circ} \mathrm{C}$ & 280.0 & 6,77 & 3 & 23 & 278.3 & 6,79 \\
\hline 7 & 25 & $285 \cdot 5$ & 6,87 & 7 & 26 & 282.7 & 6.80 \\
\hline II & 25 & 289.7 & 6,94 & II & 26 & $289 \cdot 2$ & 6,9 I \\
\hline $\mathbf{r}_{5}$ & $\downarrow$ & $\downarrow$ & $\leftarrow$ & I5 & 25 & 286.9 & 6,89 \\
\hline I9 & 22 & $292 \cdot 5$ & 7,04 & 19 & 25 & 294.0 & 7,01 \\
\hline 23 & 22 & 290.1 & 6,99 & 23 & 23 & 294.4 & 7,05 \\
\hline 27 & 23 & 288.7 & 6,95 & & & & \\
\hline $3^{I}$ & 24 & 285.5 & 6,88 & & & & \\
\hline 35 & 24 & & 6,78 & & & & \\
\hline \multicolumn{4}{|c|}{ Fall. 2.} & \multicolumn{4}{|c|}{ Fall. 4.} \\
\hline $\mathrm{D}$ & $\mathrm{T}$ & Millivolt & $\mathrm{PH}$ & $\mathrm{D}$ & $\mathrm{T}$ & Millivolt & $\mathrm{PH}$ \\
\hline 3 & $23 \cdot$ & 280.2 & $6,8 \mathrm{I}$ & 3 & 26 & $277 \cdot 2$ & $6,8 \mathrm{I}$ \\
\hline 7 & 23 & 282.1 & 6,84 & 7 & I8 & 282.0 & 6,92 \\
\hline I I & 23 & 283.2 & 6,86 & I I & 18 & 276.6 & 6,83 \\
\hline 15 & 24 & $284 \cdot 5$ & 6,87 & I5 & I8 & $285 . \mathrm{I}$ & 6,98 \\
\hline I9 & 24 & 286.8 & 6,90 & I9 & 24 & 293.0 & 7, OI \\
\hline 23 & 24 & 287.8 & 6,92 & 23 & 24 & 293.0 & $7, \mathrm{OI}$ \\
\hline 27 & 24 & 193,3 & $7, \mathrm{OI}$ & 27 & 24 & 294.7 & 7,04 \\
\hline $3^{I}$ & 25 & $295 \cdot 4$ & 7,03 & $3^{I}$ & 24 & $294 \cdot 4$ & 7,03 \\
\hline 35 & 25 & 293.9 & 7,01 & 35 & 23 & 298.8 & $7, x_{3}$ \\
\hline
\end{tabular}




\begin{tabular}{|c|c|c|c|c|c|c|c|}
\hline \multicolumn{4}{|c|}{ Fall. 5.} & \multicolumn{4}{|c|}{ Fall. 8.} \\
\hline $\mathrm{D}$ & $\mathrm{T}$ & Millivolt & $\mathrm{PH}$ & $\mathrm{D}$ & $\mathrm{T}$ & Millivolt & $\mathrm{PH}$ \\
\hline 3 & 18 & 278.2 & 6,86 & 3 & 25 & 256.4 & 6,38 \\
\hline 7 & 18 & 278.7 & 6,87 & 7 & 24 & 260.2 & 6,45 \\
\hline II & 18 & 278.3 & 6,86 & II & 22 & 264.4 & 6,55 \\
\hline 15 & $2 \mathrm{I}$ & 282.2 & 6,88 & 15 & 22 & $273 \cdot 1$ & $6,7^{\circ}$ \\
\hline 19 & 19 & 286.5 & 6,98 & 19 & $2 I$ & 279.2 & 6,82 \\
\hline 23 & $2 \mathrm{I}$ & 289.0 & 6,99 & 23 & 22 & 282.8 & 6,87 \\
\hline 27 & 21 & 287.1 & 6,96 & 27 & $2 I$ & 286.3 & 6,95 \\
\hline $3^{I}$ & $2 \mathrm{I}$ & 292.2 & 7,05 & $3^{I}$ & $2 \mathrm{I}$ & 287.6 & 6,97 \\
\hline 25 & 23 & 293.0 & 7,03 & & & & \\
\hline \multicolumn{4}{|c|}{ Fall. 6.} & \multicolumn{4}{|c|}{ Fall. 9.} \\
\hline $\mathrm{D}$ & $\mathrm{T}$ & Millivolt & PH & $\mathrm{D}$ & $\mathrm{T}$ & Millivolt & $\mathrm{PH}$ \\
\hline 3 & 19 & 257.3 & 6,48 & 3 & 25 & $276 \cdot 3$ & 6,66 \\
\hline 7 & 22 & 265.2 & 6,42 & 7 & 25 & 277.9 & $6,7+$ \\
\hline II & 23 & $273 \cdot 1$ & 6,69 & I I & 23 & 278.8 & 6,78 \\
\hline 15 & 23 & 279.8 & 6,80 & 15 & 22 & 283.0 & 6,87 \\
\hline I9 & 22 & $28 \mathrm{I} \cdot 5$ & 6,85 & 19 & 23 & 281.2 & 6.83 \\
\hline 23 & 22 & 285.2 & $6,9 \sqrt{1}$ & 23 & $\downarrow$ & $\downarrow$ & $\downarrow$ \\
\hline \multirow[t]{3}{*}{27} & 23 & 286.3 & $6,9 \mathbf{I}$ & 27 & 24 & 270.6 & 6,63 \\
\hline & & & & $3^{I}$ & 23 & 257.8 & 6.43 \\
\hline & & & & 35 & $2 \mathrm{I}$ & 253.0 & 6,37 \\
\hline \multicolumn{4}{|c|}{ Fall. 7.} & \multicolumn{4}{|c|}{ Fall. го. } \\
\hline D & $\mathrm{T}$ & Millivolt & PH & $\mathrm{D}$ & $\mathrm{T}$ & Millivolt & PH \\
\hline 3 & 23 & 252.3 & 6,33 & 3 & 26 & 263.6 & 6,48 \\
\hline 7 & 23 & 261.0 & 6,48 & 7 & 26 & 265.5 & $6,5 \mathrm{I}$ \\
\hline I I & 22 & $27 \mathrm{I} .2$ & 6,67 & II & 26 & ${ }^{\circ} 269.2$ & 6,57 \\
\hline 15 & & & & 15 & 25 & 272.4 & 6,64 \\
\hline 19 & $\downarrow$ & $\downarrow$ & $\downarrow$ & I9 & 24 & 277.0 & 6,74 \\
\hline 23 & 22 & 280.2 & 6,82 & 23 & 25 & 280.0 & 6,77 \\
\hline 27 & 22 & $28 \mathrm{I} .6$ & 6,85 & 27 & 24 & 282.5 & 6,83 \\
\hline $3^{I}$ & 22 & 283.4 & 6,88 & $3^{I}$ & 23 & 289.8 & 6,97 \\
\hline 35 & 24 & 288.2 & 6,93 & 35 & 24 & 292.5 & 7,00 \\
\hline
\end{tabular}


Die Bestimmung der Wasserstoffionenkonzentration in der Oto-Rhino-Laryngologie. ; 959

\begin{tabular}{|c|c|c|c|c|c|c|c|}
\hline \multicolumn{4}{|c|}{ Fall. Ir. } & \multicolumn{4}{|c|}{ Fall. I4. } \\
\hline $\mathrm{D}$ & $\mathrm{T}$ & Millivolt & $\mathrm{PH}$ & $\mathrm{D}$ & $\mathrm{T}$ & Millivolt & $\mathrm{PH}$ \\
\hline 3 & 22 & 270.1 & 6,65 & 3 & 23 & 254.5 & 6,37 \\
\hline 7 & 23 & 271.8 & 6,67 & 7 & 23 & $26 \mathrm{I} \cdot 5$ & 6,49 \\
\hline I I & 22 & 276.2 & 6,76 & II & 23 & 275.2 & 6,72 \\
\hline 15 & 22 & 278.3 & 6,79 & I 5 & 24 & 267.0 & 6,57 \\
\hline 19 & 24 & 282.5 & 6,83 & 19 & 24 & 285.7 & 6,89 \\
\hline 23 & 24 & 285.4 & 6,88 & 23 & 24 & $282 . \mathrm{I}$ & 6,82 \\
\hline $2 \tau$ & 22 & 288.2 & 6,96 & 27 & 23 & 291.0 & 6,99 \\
\hline $3 I$ & $\downarrow$ & $\downarrow$ & $\downarrow$ & $3^{\mathbf{T}}$ & 22 & 299.9 & 7,14 \\
\hline 35 & 23 & $29 \mathrm{I} .4$ & 7,00 & 35 & 24 & 298.5 & 7,10 \\
\hline \multicolumn{4}{|c|}{ Fall. 12.} & \multicolumn{4}{|c|}{ Fall. I5. } \\
\hline D & $\mathrm{T}$ & Millivolt & PH & $\mathrm{D}$ & $\mathrm{T}$ & Millivolt & $\mathrm{PH}$ \\
\hline 3 & 24 & 272.4 & 6,66 & 3 & 22 & $275 \cdot 5$ & 6,74 \\
\hline 7 & 24 & $275 \cdot 2$ & $6,7 \mathrm{I}$ & 7 & 22 & 278.2 & 6,79 \\
\hline II & 24 & 280.4 & 6,80 & I I & 22 & 285.4 & $6,9 \mathrm{I}$ \\
\hline 15 & 25 & 279.5 & 6,76 & 15 & 23 & 283.2 & 9,86 \\
\hline \multirow[t]{5}{*}{19} & 23 & 282.6 & 6,85 & 19 & $\downarrow$ & $\downarrow$ & $\downarrow$ \\
\hline & & & & 23 & 24 & 296.0 & 7,06 \\
\hline & & & & 27 & 22 & 292.9 & $7,0_{4}$ \\
\hline & & & & $3 I$ & 22 & 298.5 & $7, \mathbf{1} 4$ \\
\hline & & & & 35 & 23 & 302.4 & 7,19 \\
\hline \multicolumn{4}{|c|}{ Fall. I3. } & \multicolumn{4}{|c|}{ Fall. I6. } \\
\hline $\mathrm{D}$ & $\mathrm{T}$ & Millivolt & $\mathrm{PH}$ & $\mathrm{D}$ & $\mathrm{T}$ & Millivolt & $\mathrm{PH}$ \\
\hline 3 & 25 & 274.0 & 6,67 & 3 & 24 & 282.7 & 6,83 \\
\hline 7 & 25 & 286.7 & 6,89 & 7 & 24 & $284 \cdot 4$ & 6,86 \\
\hline II & 25 & 286.3 & 6,88 & II & 24 & 289.4 & 6,95 \\
\hline 15 & 23 & $291 \cdot 3$ & 7,00 & I 5 & 25 & 29I.I & 6,96 \\
\hline 19 & 24 & 288.0 & 6,92 & 19 & 24 & $295 \cdot 5$ & 7,05 \\
\hline \multirow[t]{4}{*}{23} & 25 & 288.4 & $6,9 \mathrm{I}$ & 23 & 23 & 296.0 & 7,09 \\
\hline & & & & 27 & 24 & $299 \cdot 4$ & 7,12 \\
\hline & & & & $3 I$ & 23 . & 300.8 & 7,16 \\
\hline & & & & 35 & 23 & 304.9 & 7,23 \\
\hline
\end{tabular}




\begin{tabular}{|c|c|c|c|c|c|c|c|}
\hline \multicolumn{4}{|c|}{ Fall. I7. } & \multicolumn{4}{|c|}{ Fall. 20.} \\
\hline $\mathrm{D}$ & $\mathrm{T}$ & Millivolt & $\mathrm{PH}$ & $D$ & $\mathrm{~T}$ & Millivolt & $\mathrm{PH}$ \\
\hline 3 & 24 & $264 \cdot I$ & 6,52 & 3 & 25 & 277.8 & 6,74 \\
\hline 7 & 24 & 270.4 & 6,63 & 7 & 25 & 278.8 & 6,75 \\
\hline II & 24 & 267.2 & 6,57 & II & 25 & 280.2 & 6,78 \\
\hline \multirow[t]{6}{*}{15} & 25 & 273.0 & 6,65 & 15 & 25 & 280.2 & 6,78 \\
\hline & & & & I9 & 24 & 281.I & $6,8 \mathrm{I}$ \\
\hline & & & & 23 & 24 & 282.0 & 6,82 \\
\hline & & & & 27 & 24 & 282.6 & $6,8_{3}$ \\
\hline & & & & $3^{I}$ & $\downarrow$ & $\downarrow$ & $\downarrow$ \\
\hline & & & & 35 & 24 & 283.5 & 6,85 \\
\hline \multicolumn{4}{|c|}{ Fall. I8. } & \multicolumn{4}{|c|}{ Fall. $2 \mathrm{r}$. } \\
\hline $\mathrm{D}$ & $\mathrm{T}$ & Millivolt & $\mathrm{PH}$ & $\mathrm{D}$ & $\mathrm{T}$ & Millivolt & $\mathrm{PH}$ \\
\hline 3 & 25 & 259.9 & 6,43 & 3 & 25 & 261.2 & 6,45 \\
\hline 7 & 25 & 262.4 & 6,47 & 7 & 25 & 273.0 & 6,65 \\
\hline II & 24 & 265.5 & 6,54 & I I & 25 & 276.3 & $6,7 \mathrm{I}$ \\
\hline 15 & 25 & $267 \cdot 7$ & 6,56 & I5 & 25 & $279 \cdot 1$ & $6,7^{6}$ \\
\hline 19 & 24 & 272.0 & 6,65 & 19 & 24 & $28_{3} \cdot 0$ & 6,84 \\
\hline 23 & 23 & $273 \cdot 1$ & 6,69 & 23 & 24 & 284.6 & 6,87 \\
\hline 27 & 24 & 275.8 & 6,72 & 27 & 24 & 288.5 & $6,9.3$ \\
\hline $3^{I}$ & 25 & 276.8 & 6,72 & & & & \\
\hline 35 & 25 & 278.8 & 6,75 & & & & \\
\hline \multicolumn{4}{|c|}{ Fall. I9. } & \multicolumn{4}{|c|}{ Fall. 22.} \\
\hline $\mathrm{D}$ & $\mathrm{T}$ & Millivolt & $\mathrm{PH}$ & $\mathrm{D}$ & $\mathrm{T}$ & Millivolt & $\mathrm{PH}$ \\
\hline 3 & 24 & $256 . \mathrm{I}$ & $6,3^{8}$ & 3 & 24 & 270.6 & 6,63 \\
\hline 7 & 24 & $259 \cdot 5$ & 6,44 & 7 & 25 & 272.2 & 6,64 \\
\hline I I & 24 & $26_{3} .0$ & 6,50 & I I & 24 & 273.5 & 6,68 \\
\hline 15 & 24 & $264 \cdot 2$ & $5,5^{2}$ & $\mathbf{I} 5$ & 24 & 276.6 & 6,73 \\
\hline 19 & 24. & 263.9 & 6,62 & 19 & 24 & $277 \cdot 7$ & 6,75 \\
\hline 23 & 23 & 270.0 & 6,62 & 23 & 24 & 279.0 & 6,77 \\
\hline 27 & 24 & 278.7 & 6,77 & 27 & 24 & 282.7 & 6,83 \\
\hline $3^{1}$ & 24 & $275 \cdot 3$ & $6,7 \mathrm{I}$ & $3 I$ & 23 & 286.0 & $6,9 \mathbf{I}$ \\
\hline 35 & 24 & 278.7 & 6,77 & 35 & 24 & 290.0 & $6,9^{6}$ \\
\hline
\end{tabular}


Die Bestimmung der Wasserstoffionenkonzentration in der Oto-Rhino-Iaryngologie. 361

Tabelle 3.

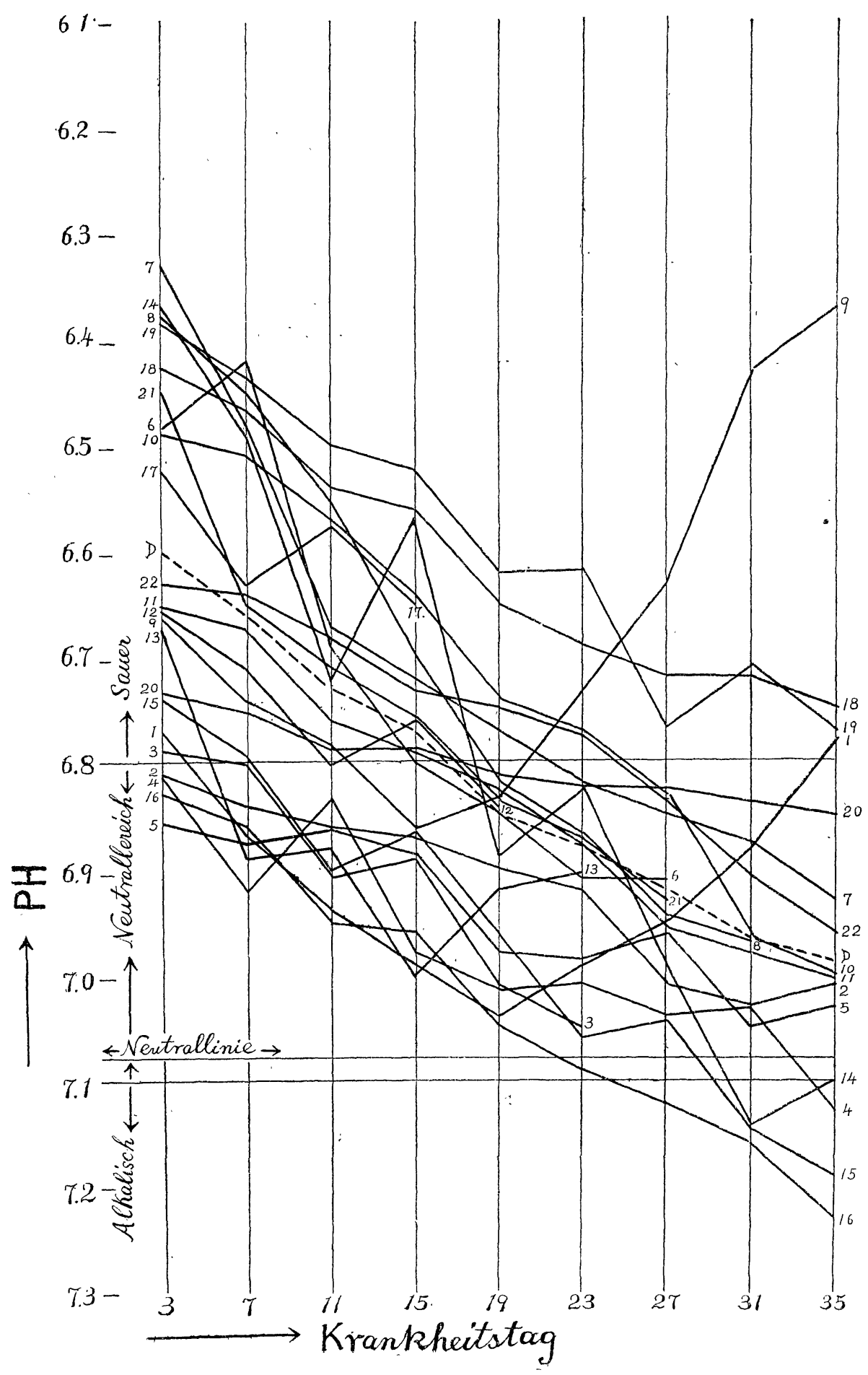

